\section{Zwischen Überleben und Gutem Leben}

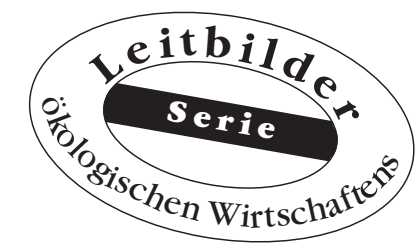

Nachhaltigkeit ist ein ziemlich kompliziertes und zudem ein relativ dröges Leitbild. Mit seinen drei Dimensionen bietet es zwar große Chancen für historische Kompromisse und für die Auflösung bisheriger Grabenkämpfe. Die Anforderung einer permanenten gleichwertigen Berücksichtigung der ökologischen, sozialen und ökonomischen Dimension birgt aber auch die Gefahr, dass gar keine Schritte mehr gewagt werden.

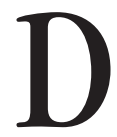
Von Arnim von Gleich ie Versuchung, in der Nachhaltigkeitsdiskussion bei allgemeinem Geschwafel stehen zu bleiben, ist offensichtlich groß. Das hat mehrere Gründe, und die sind nicht nur bei der allgemeinen Öffentlichkeit zu suchen, die noch nicht einmal den Begriff richtig kennt, sondern gerade auch bei den Protagonisten ökologischer, sozialer und ökonomischer Anliegen. Sich auf das Ziel Nachhaltigkeit richtig einzulassen, erfordert erstens gewaltige innere Umorientierungen. Genau besehen verstehen derzeit aber die meisten Akteure Nachhaltigkeit nach wie vor als die Fortsetzung ihres bisherigen Umwelt-, Sozial-, Dritte Welt- und Wirtschaftsengagements unter einem neuen Banner. Das Neue am Nachhaltigkeitsthema ist aber nicht nur die Dreidimensionalität, sondern vor allem die Langfristorientierung und (nicht ganz so neu) die globale Orientierung, also eine gewaltige Ausdehnung unserer Problemwahrnehmung in Raum und Zeit. Dieser Ausdehnung der Problemwahrnehmung folgt aber zweitens nicht automatisch eine Ausdehnung unserer Problemlösungskapazitäten.

Drittens ist Nachhaltigkeit eher ein defensives Leitbild, mehr am Überleben als am „Guten Leben“ orientiert. Dies liegt nicht nur an der Dominanz der ökologisch-ökonomischen RioThemen Klima und Ressourcen sowie Biodiversität. Selbst das eigentliche Kernthema des Nachhaltigkeitsdiskurses, die Gerechtigkeit, bietet - verstanden als Verteilungs-Gerechtigkeit zwischen Industrienationen und Entwicklungs- und Schwellenländern sowie zwischen heute lebenden und zukünftigen Generationen - für die Bewohner der Industrienationen mehr Zumutungen als Hoffnungen.

\section{Leitbilder oder Leitplanken}

Strategien zur Umsetzung des Leitbildes Nachhaltigkeit kann man zwei Idealtypen zuordnen, dem Steuerungsmodell und dem Prozessmodell. Das Steuerungsmodell steht für eine defensive 0perationalisierung. Nachhaltige Entwicklung wird verstanden als ein Weg in die Zukunft, bei dem zumindest große Crashs in den ökologischen, sozialen und ökonomischen Systemen vermieden werden. Hier geht es also vor allem ums Überleben. Crashvermeidung erfordert aber ein Wissen über Tragekapazitäten, auf dessen Basis dann Leitplanken für zukünftige Entwicklungskorridore formuliert werden können. Die Durchsetzung und Sicherung dieser Rahmenbedingungen ist dann, ganz im Sinne der Gefahrenabwehr, eine staatliche Aufgabe.

Nehmen wir das Beispiel Klimapolitik. Hier hat der Wissenschaftliche Beirat der Bundesregierung Globale Umweltveränderungen (WGBU) als Leitplanken das Kontingent an Treibhausgasen errechnet, das in den kommenden 100 Jahren höchstens global zusätzlich zum natürlichen Treibhauseffekt emittiert werden darf, wenn die Anpassungsfähigkeit der ökologischen, sozialen und ökologischen Systeme gewährleistet bleiben soll. Umweltverbände und Dritte-Welt-Gruppen sind weiter gegangen und haben - im Sinne einer formalen Verteilungsgerechtigkeit - diese Menge durch die Zahl der Weltbevölkerung geteilt. Sie kamen damit zu jenen 2,3 Tonnen Treibhausgasen (in KohlendioxidÄquivalenten) pro Kopf und Jahr, die zu den gegenwärtigen 9 bis 15 Tonnen pro Kopf und Jahr in den Industrieländern doch stark kontrastieren. Aus solchen Abschätzungen folgen somit recht harte Leitplanken. Sie bilden die Basis für Forderungen wie jener; den Stoff- und Energieumsatz in den In- dustrieländern um den Faktor 10 zu reduzieren. Dann bleibt ,nur" noch die Aufgabe für die Staaten, solche Margen mit Hilfe von Klimarahmenkonventionen, Ökosteuern, Tempolimits, Wärmeschutzverordnungen, freiwilligen Selbstverpflichtungen und dergleichen umzusetzen. Was sein muss, muss sein.

Das Prozessmodell steht dagegen für eine offensive Operationalisierung. Das Leitbild Nachhaltigkeit muss für verschiedene Bedürfnis- und Handlungsfelder konkretisiert werden. An Runden Tischen werden Konzepte für eine nachhaltige Mobilität, für nachhaltiges Bauen und Wohnen, für eine nachhaltige Region oder Kommune, für eine nachhaltige Hochschule oder für ein nachhaltiges Unternehmen erarbeitet. Hier wäre Platz für begeisternde Zukunftsentwürfe. Auf all diesen Feldern könnten tatsächlich mindestens drei verschiedenePfade in die Zukunft entworfen werden:

- Crash-Pfade als Trendszenarien, wenn alles so weiter läuft wie bisher,

- ein Crashvermeidungs- bzw. Überlebenspfad mit den nötigen Umsteuerungen (hier könnte man durchaus an den schon vorhandenen, wenn auch zum Teil noch stark technikfixierten Energie-, Verkehrs- und Chemiewenden anknüpfen) und schließlich

- Pfade in Richtung gutes Leben, gutes Wohnen oder in Richtung Zugang und Ankommen als Alternative zu rastloser Mobilität.

\section{Legitimationsprobleme}

Beide Strategievarianten, das Steuerungs- und das Prozessmodell, sind mit je spezifischen Problemen behaftet; die meisten haben etwas mit Legitimation zu tun. Das Steuerungsmodell ist auf Wissen über Tragekapazitäten von ökologischen, sozialen und ökonomischen Systemen angewiesen, womit die Wissenschaft, die im Steuerungsmodell neben dem Staat die Hauptrolle spielt, stark überfordert ist. Bisher konnten - mit Hilfe gewaltiger Abstraktionen - selbst im schon lange bearbeiteten ökologischen Bereich nur Tragekapazitäten bzw. „,critical loads“ für den Eintrag versauernder und eutrophierender Substanzen in ökosysteme sowie für klimarelevante und ozonzerstörende Gase formuliert werden. Über Tragekapazitäten von sozialen und ökonomischen Systemen ist fast nichts bekannt. Dies könnte auch so bleiben, weil verlässliche Prognosen über die Folgen von Eingriffen in komplexe und dynamische Systeme prinzipiell unmöglich sind.

Wenn die Gesellschaft solche wissenschaftlichen Aussagen, wie jetzt beispielsweise zur Klimapro- 
blematik „für wahr“ nimmt und daraus weitreichende Konsequenzen ableitet, lädt sich die Wissenschaft zudem eine ungeheure Verantwortung auf. Im Moment sonnen sich die Wissenschaftler noch in dieser neuen Aufmerksamkeit. Das kann aber auch gewaltig schief gehen, mit noch tiefgreifenderen Legitimationsverlusten, als wir sie schon vom Spiel ,Gutachten gegen Gegengutachten“ aus den vergangenen Jahrzehnten kennen. Zudem transportiert das Steuerungsmodell mit seinem „Wissenschaft gibt vor und Politik setzt um" ein ziemlich unmodernes etatistisches kausal-mechanisches Politikverständnis. Auch dies dürfte im Zuge des beobachtbaren Verlusts an Steuerungsmöglichkeiten staatlicher Politik zu weiteren Frustrationen führen.

Das Prozessmodell erscheint demgegenüber moderner. Hier wird schließlich auf Dezentralität und auf zivilgesellschaftliche Akteure gesetzt. Während aber die Legitimation politischer und staatlicher Akteure im Steuerungsmodell durch Wahlen einigermaßen fundiert ist, kämpfen die zivilgesellschaftlichen Akteure im Prozessmodell mit großen Legitimationsproblemen. Woher beziehen die Teilnehmer an den Runden Tischen ihre Legitimation, und wie erreichen sie die Verbindlichkeit ihrer Beschlüsse? Die wenigsten Schritte in Richtung Nachhaltigkeit dürften win-win-win-Schritte im Sinne des dreidimensionalen Nachhaltigkeitsziels sein. Wer sorgt für Verbindlichkeit oder zumindest personelle Kontinuität? Wer sorgt dafür, dass ein Anliegen, das diesmal zurück stecken musste, beim nächsten Mal zum Zuge kommt? Gibt es hier die Möglichkeit für eine „Legitimation durch Verfahren" wie sie in der Wissenschaft oder in der Rechtsprechung funktioniert, oder reicht eine Einbezie- hung von Wissenschaft und Politik in den Diskurs, wie dies etwa in Enquete-Kommissionen der Fall ist? Wenn das Prozessmodell ein Erfolgsmodell werden soll, sind hier jede Menge institutionelle Innovationen gefragt.

\section{Integration durch Leitbilder?}

In säkularisierten und ausdifferenzierten, ja geradezu fragmentierten modernen Gesellschaften könnten und sollten Leitbilder integrierend, orientierend und motivierend wirken (vgl. Zundel in Ökologisches Wirtschaften 2/2000). Womöglich sind das aber sich widersprechende Anforderungen. Die Wahrscheinlichkeit, dass die motivierende Funktion von konkreten Utopien (positiven Lebensentwürfen) Subkulturen übergreifend funktioniert, wird immer geringer. Ist es heute noch sinnvoll möglich, ein Leitbild nachhaltige Mobilität oder nachhaltiges Bauen und Wohnen für alle zu entwerfen? Würde der Nachhaltigkeitsdiskurs damit nicht tatsächlich zur Waffe des nationalen und internationalen Kulturimperialismus, als die ihn einige Dritte-WeltGruppen ohnehin schon geißeln?

Allem Anschein nach besteht diese Gefahr aber gar nicht, weil Utopien und Vorstellungen vom Guten Leben im Nachhaltigkeitsdiskurs ohnehin kaum eine Rolle spielen. Gruppenübergreifende Einigungen scheinen am ehesten auf dem kleinsten gemeinsamen Nenner möglich zu sein, und der lässt sich leichter defensiv formulieren. Ohnehin geht derzeit die gesellschaftliche Dynamik nicht von unseren normativen Anliegen aus. Wir werden eher von der Modernisierungswelle getrieben als vom Leitbild angezogen. Doch kann das so bleiben? Die Kraft für Veränderungen wird Ernst Bloch zufolge aus dem „Wärmestrom“ geschöpft, der von „,konkreten Utopien“ ausgeht. Wenn das Nachhaltigkeitsziel motivierende Kraft entfalten soll, führt an ihnen womöglich kein Weg vorbei. Es könnte aber sein, dass solche konkreten Utopien heute nur noch gruppenspezifisch formuliert werden können. Die Integration in ein einziges großes Nachhaltigkeitsleitbild wäre dann kaum möglich.

Auch im Prozessmodell scheint sich somit eher die Leitplanke als das Leitbild durchzusetzen. Vieles spricht dafür, dass sich das ohne Zweifel große integrative Potential des dreidimensionalen Leitbilds Nachhaltigkeit auf die Sicherung der Entwicklungsfähigkeit der Gesellschaft insgesamt (und damit auch der ganzen verschiedenen Gruppen) beschränkt und auf die Formulierung von Mindestvoraussetzungen konzentriert. Im Nachhaltigkeitsdiskurs werden weiterhin eher Vor-Sorge-Motive, als motivierende Utopien dominieren. Die Verbindung $\mathrm{zu}$ den gruppen- und handlungsfeldspezifischen Leitbildern bleibt prekär. Vorsorge für das bloße Überleben geschieht aus Einsicht in die Notwendigkeit. Das reißt niemanden wirklich vom Hocker. „No risk no fun!“ ist schließlich nicht völlig von der Hand zu weisen.

\section{Der Autor}

Dr. Arnim von Gleich ist Professor an der Fachhochschule Hamburg.

Konfakt: FH Hamburg, FB Maschinenbau und Produktion, Berliner Tor 21, 20099 Hamburg, Tel. 040/ 42859-4345, Fax -2658,

E-mail: gleich@rzbt.fh-hamburg.de

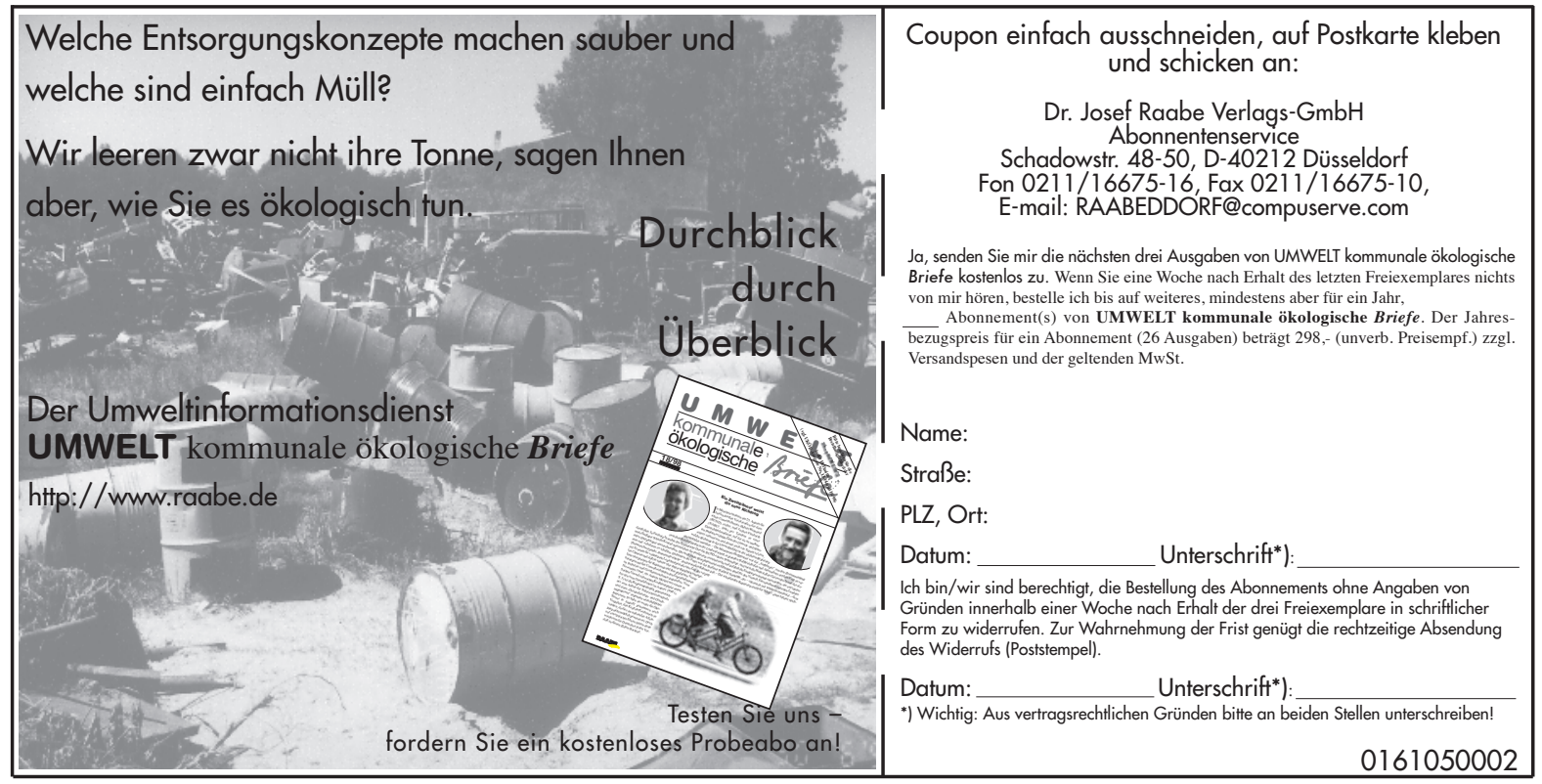


(c) 20I0 Authors; licensee IÖW and oekom verlag. This is an article distributed under the terms of the Creative Commons Attribution Non-Commercial No Derivates License (http://creativecommons.org/licenses/by-nc-nd/3.o/), which permits unrestricted use, distribution, and reproduction in any medium, provided the original work is properly cited. 